\title{
Two-Dimensional Phase Unwrapping with Continuous Submodular Minimization
}

\author{
Hiroyuki Kudo \\ University of Tsukuba \\ 1-1-1 Tennodai \\ Tsukuba, Ibaraki, Japan \\ kudo@cs.tsukuba.ac.jp
}

\author{
Songzhe Lian \\ University of Tsukuba \\ 1-1-1 Tennodai \\ Tsukuba, Ibaraki, Japan \\ songzhe.lian@gmail.com
}

\author{
Katsuhiro Wada \\ University of Tsukuba \\ 1-1-1 Tennodai \\ Tsukuba, Ibaraki, Japan
}

\begin{abstract}
The phase unwrapping is recovering true phase from its $2 \pi$ modulo observations which are related to some discrete optimization problems. The challenge is to exactly solve the discrete optimization problem arising from noisy data. In this paper, we propose a new continuous minimization method for phase unwrapping. Using the Lovász extension we transform the discrete problem to equivalent continuous problem. In contrast to conventional continuous minimization methods, our method can solve this discrete optimal problem exactly. In addition, one regularization term is added to the energy function to deal with noisy images. By using $L^{1}$ norm for both data term and regularization term our method performs well for discontinuous images. Moreover, its implementation is very simple. A set of experiment results illustrates the effectiveness of the proposed method.
\end{abstract}

\section{CCS Concepts}

- Theory of computation $\rightarrow$ Design and analysis of algorithms $\rightarrow$ Mathematical optimization $\rightarrow$ Mixed discrete-continuous optimization $\rightarrow$ Submodular optimization and polymatroids

- Computing methodologies $\rightarrow$ Computer graphics $\rightarrow$ Image manipulation $\rightarrow$ Image processing

\section{Keywords}

Phase unwrapping; Lovász extension; Regularization term; Minimum norm

\section{INTRODUCTION}

Phase unwrapping is the process of attempting to reconstruct the true phase from modulo $2 \pi$ phase values. It is motivated by a variety of applications. Interferometric synthetic aperture radar (InSAR) uses two or more radars to form maps of deformation for an object which is applied into geodesy and remote sensing [1], [2]. In X-ray phase imaging, phase shift caused by the object is not measured directly, but resulted in some variations in intensity

Permission to make digital or hard copies of all or part of this work for personal or classroom use is granted without fee provided that copies are not made or distributed for profit or commercial advantage and that copies bear this notice and the full citation on the first page. Copyrights for components of this work owned by others than the author(s) must be honored. Abstracting with credit is permitted. To copy otherwise, or republish, to post on servers or to redistribute to lists, requires prior specific permission and/or a fee. Request permissions from Permissions@acm.org.

DMIP'18, November 12-14, 2018, Okinawa, Japan

(C) 2018 Copyright is held by the owner/author(s). Publication rights licensed to ACM.

ACM ISBN 978-1-4503-6578-9/18/11 ..\$15.00

DOI: https://doi.org/10.1145/3299852.3299864 images. By recovering the phase shift, the internal structure of biological soft tissues can be observed which is difficult for conventional X-ray CT [3], [4]. Phase unwrapping is also useful for water and fat separation problem in MRI [5]. In these applications, the phase information of object is included in some periodic signals. Then the phase is determined only in the principal value range of $[-\pi, \pi)$ as shown in Figure 1. It is called as phase unwrapping- recovering true phases with wrapped ones.

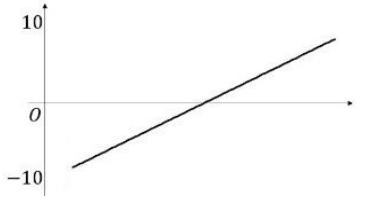

(a)

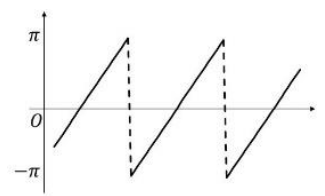

(b)
Figure 1. (a) Continuous phase (b) Wrapped phase

In general, without additional conditions it is impossible to recover the true phase exactly from the measured signal that is affected by noise and is wrapped into principle value range. And the most common assumption is that the gradient (differences between neighboring pixels) of unwrapped phases is less than $\pi$ (Itoh condition) [6]. If this assumption is valid in every pixel, unwrapping is simply completed by the path-following method as following.

We can start at an arbitrary point of arbitrary path to plus (or minus) some integer multiple of $2 \pi$ for each pixel such that the phase differences between neighboring pixels are less than $\pi$. Then unwrapping is completed while those paths cover the entire image.

In practical applications, Itoh condition might fail. It can be caused by the discontinuity in the true phase or noise. Then for $2 \mathrm{D}$ phase unwrapping, different paths may result in different unwrapped phase values for one pixel. It means a closed loop exists there with non-zero line integrals which is known as the residue. To solve the path depending problem, several techniques with path-following methods have been developed such as branch cuts and minimum cost flow (MCF) etc. [7]-[9].

In branch cuts, all residues are computed firstly by searching all the smallest closed paths i.e. $2 \times 2$ sample box. Then define cut lines by finding pairings of neighboring opposite residues. It will give unique unwrapped phase image by prevent paths crossing these cut lines [9].

Flynn proposed minimum cost flow method that also defines the path using the positive and negative residues. Different with branch cuts it detects the discontinuities point by point to determine the path explicitly. These discontinuous points are 
decreased by plus (or minus) integer multiple of $2 \pi$ along the path. Then discontinuity is minimized by iteration. This method can reach a global optimal solution and works robustly in many cases with low noisy image.

However, the counts of pixels with non-zero residue will increase rapidly for the high level noisy images. Then for path-following methods, it is not obvious to find the best path or exact cut lines, and it makes unwrapping difficult.

Another important development is minimum norm (energy minimization) method which is path independent. These methods try to find the unwrapped phase image by minimizing the difference between the gradient of the true phase and that of the wrapped phase according to a $L^{p}$ norm. For $\mathrm{p}=2$, it is least squares problem and can be solved using the fast Fourier transform [9]. For general p, this problem is related to a partial differential equation (LP-PDE) by calculus of variations and it is solved using weighted least-squares algorithms [10]. Similar technique is developed to unwrapping 4D MR flow data [11]. In addition, some techniques with regularization terms are also developed for the wrapped signal with high level noise [12], [13]. These path independent methods aim the global optimal solution and are more robust for the noisy image than path-following methods generally. More details on typical minimum norm methods will be given in the following section.

Note that there is an important congruent constraint in phase unwrapping i.e.

Rewrapping unwrapped image should be identical to the original wrapped image.

(CC)

Strictly speaking, the (CC) condition causes a discrete optimization problem. Most minimum norm methods is to solve continuous minimization problem directly and leading the solution to fail the congruent test. This difficulty is solved by using the Graph cuts method which is currently considered state of the art [14]. Note that the Graph cuts method needs to increase memory usage for additional variables such as node and it is related to the techniques such as construction of Graph and network flow etc. It is not easy extends the method to the higher dimension problem which is related to more complex Graph and which force us to increase memory usage rapidly [15-17].

In this paper, we propose a new path independent method with continuous submodular minimization. Its derivation is outlined as follows. First we define an energy function consisting of data term and weighted regularization term. Then transform the minimization problem from the discrete optimization to equivalent continuous one using Lovász extension [18, 19]. Note that after extension we will have a non-differential function to minimize. Therefore, the double averaging subgradient method was used [20].

Our contribution consists of three parts.

a) Solve a continuous minimization problem and reach optimal solution with $(\mathbf{C C})$ condition which is the first result for phase unwrapping.

b) It is robust for noisy images by additional L1 norm regularization term. Note that the regularization term lets adjacent points have the same $2 \pi$ jump as much as possible to effectively act as a denoising process. But unlike usual denoising technique it can preserve the $(\mathbf{C C})$ condition because it changes unwrapped function by only integer multiple of $2 \pi$.

c) Unlike previous network flow methods it does not need to increase additional memory usage. Moreover, its way of implementation is very simple and can be extended to the $3 \mathrm{D}$ phase unwrapping problem easily.

This paper is organized as follows. In section 2, we review existing typical minimum norm methods. In section 3 , we derive the proposed method. In section 4 , we show several experiment results. The conclusion is given in section 5 .

\section{ENERGY MINIMIZATION}

In this subsection, we review existing typical methods with energy minimization.

In many applications, phases are extracted from both sin and cos signal using an extended arctangent functions that give the wrapped phase. And then we need to recover the true phase from the wrapped phase. Formally, we have

$$
\psi_{i, j}=\varphi_{i, j}-2 \pi d_{i, j} \stackrel{\operatorname{def}}{=} W\left(\varphi_{i, j}\right),(i, j) \in[0, M-1] \times[0, N-1],
$$

where $l_{i, j}$ is integer, $\varphi_{i, j}$ and $\psi_{i, j}$ are the true phase and wrapped phase respectively. The wrapping operator $W(\cdot)$ maps functions into the principal value range $[-\pi, \pi)$.

\subsection{Energy Function}

In path following methods, the unwrapping is completed to plus (or minus) integer multiple of $2 \pi$ pixel by pixel for wrapped image. Different with it most minimum norm methods to deal with difference function directly for both unwrapped phase and wrapped phase. Denote horizontal and vertical direction difference by

$$
\begin{array}{ll}
\Delta^{h} \psi_{i, j}=\psi_{i+1, j}-\psi_{i, j}, & i<M-1, \\
\Delta^{v} \psi_{i, j}=\psi_{i, j+1}-\psi_{i, j}, & j<N-1,
\end{array}
$$

respectively.

In the following with one dimensional example we explain how to define a suitable energy function. Consider one wrapped function that is formed using wrapping operation for one continuous function as in Figure 1. From its difference image Figure 2. (a), we find that the absolute value of difference function in wrapped point is bigger than other points. Hereby we can determine the map of wrapped points from difference image. Formally

$$
\left|\Delta^{h} \psi(A)-W\left(\Delta^{h} \psi(A)\right)\right|,\left|\Delta^{h} \psi(B)-W\left(\Delta^{h} \psi(B)\right)\right|=2 \pi,
$$

and it vanishes for other points. On the other hand, for true phase, it will vanish for every point. In this sense, unwrapping is equivalent to find one integer function $l_{i}$ so that it will minimize the following problem

$$
\min _{\left\{l_{i}\right\}} \sum_{i}\left|2 \pi \Delta^{h} l_{i}+\Delta^{h} \psi_{i}-W\left(\Delta^{h} \psi_{i}\right)\right|
$$




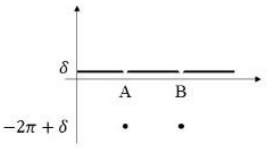

(a)

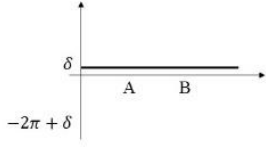

(b)

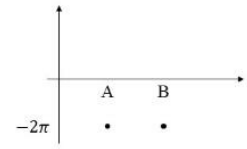

(c)
Figure 2. (a) Difference image $\Delta^{h} \psi$ (b) Wrapped difference image $W\left(\Delta^{h} \psi\right)$ (c) Second order difference $\Delta^{h} \psi-W\left(\Delta^{h} \psi\right)$

For two dimensional images, consider both horizontal and vertical direction, the energy minimization problem can be written as following

$$
\begin{gathered}
\min _{\left\{l_{i, j}\right\}} E\left(l_{i, j}\right)^{d e f}=\min _{\left\{l_{i, j}\right.} \sum_{i, j}\left(\left|\Delta^{h}\left(2 \pi d_{i, j}+\psi_{i, j}\right)-W\left(\Delta^{h} \psi_{i, j}\right)\right|^{p}\right. \\
\left.+\left|\Delta^{v}\left(2 \pi l_{i, j}+\psi_{i, j}\right)-W\left(\Delta^{v} \psi_{i, j}\right)\right|^{p}+\beta S\left(l_{i, j}\right)\right) \\
l_{i, j} \text { is integer, }
\end{gathered}
$$

where $p \geq 1$ is constant. The last term in equation (6) is the regularization term with $\beta \geq 0$ as its weight. The regularization term is added in order to prevent noises

The energy function in equation (6) takes two-dimensional information with horizontal and vertical direction simultaneously to solve the global minimum which is different with path following methods.

\subsection{Priori work}

The equation (7) comes from (CC) condition means phase unwrapping is a discrete optimization problem. It is well known that is difficult to be solved by continuous minimization methods. And many of minimum norm methods could only solve equation (6). When $p=2$, it is solved by weighted and unweighted leastsquares method respectively. In unweighted least-squares method, it is transformed to Poisson equation and can be implemented with the fast Fourier transform (FFT) at high speed. Note that, solving Poisson equation means it will give smoother solution than the true phase generally. In weighted least-squares method (PCG), the weight is added for decreasing the influence of low quality area such as high level noise areas etc. For general $p$, based on Euler-Lagrange equation, equation (6) is transformed to a nonlinear partial differential equation. Taking the nonlinear part as a weight in the iteration process, we can solve this problem. In addition, other continuous minimization methods with regularization term are solved by iteration process.

Note that these solutions could not satisfy equation (7). It is obvious that we can impose this (CC) condition after unwrapping using round up (or down) process as following

$$
l_{i, j}^{\text {new }}=\operatorname{int}\left(l_{i, j}\right) .
$$

However it raises concern that the new function may not minimize the energy function.

The Graph-cuts method can solve equation (6) and (7) simultaneously and gives good results in many applications. In the Graph-cuts method, first construct a graph with vertices and edges corresponding to each pair of adjacent points. These vertices are divided into two disjoint sets (source and sink) by a cut process. Using given energy function, we can define the weight for the graph to calculate the cost for every cut. It is important that the minimum cut on the graph also minimizes the energy function by a specialized graph construction. Then unwrapping problem is solved by finding minimum cut with max flow algorithms.

Example A Numbers of variable for graph cuts

In the Graph cuts method, the inflow and outflow for one pixel are different generally. Then we need approximately

$n$ (vertices) $+4 n$ (edges) variables where $n$ is image size (see

Figure 3.). It needs $9 n$ variables when we also use the diagonal edges. In contrast, minimum norm methods require only $n$ variables generally. It is obvious that for $3 \mathrm{D}$ problem we have to use more variables which means more memory usage and need to construct more complex graphs [15].

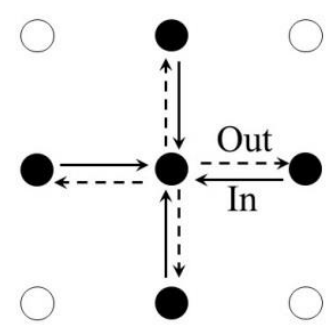

Figure 3. Variable for graph cuts

Table1. Energy minimization methods

\begin{tabular}{|c|c|c|c|c|}
\hline & $\begin{array}{c}\text { Least- } \\
\text { Squares }\end{array}$ & $\begin{array}{c}\text { LP- } \\
\text { PDE }\end{array}$ & $\begin{array}{c}\text { Gragh } \\
\text { Cuts }\end{array}$ & $\begin{array}{c}\text { Proposed } \\
\text { algorithm }\end{array}$ \\
\hline $\begin{array}{c}\text { (CC) condition } \\
\text { (exact solution) }\end{array}$ & No & No & Yes & Yes \\
\hline $\begin{array}{c}\text { More memory } \\
\text { usage }\end{array}$ & No & No & Yes & No \\
\hline
\end{tabular}

In this paper, we will propose new continuous minimization method that can solve equation (6) and (7) simultaneously and then give the exact global minimum solution.

\section{CONTINUOUS SUBMODULAR MINIMIZATION}

In this subsection we derive the proposed method. First, the Lovász extension is introduced which have been applied in many submodular optimal problems. Next, we will transform phase unwrapping problem (discrete) to equivalent continuous minimization problem. Then, the double averaging subgradient method is described to solve the minimization problem. Finally, introduce our algorithm combining these techniques.

\subsection{Lovász extension}

Lovász extension can extend a set function (or equivalent discrete function) to a continuous function which is used in integer programming etc. 
For finite set $\Omega=\{1,2, \cdots, k\}(k>1)$, consider set functions $f: 2^{\varsigma 2} \rightarrow R$ where $2^{\Omega 2}$ denote the power set of $\Omega$. We say $f$ is submodular function, if for every $T_{1}, T_{2} \subseteq \Omega$,

$$
f\left(T_{1} \cap T_{2}\right)+f\left(T_{1} \cup T_{2}\right) \leq f\left(T_{1}\right)+f\left(T_{2}\right) .
$$

Note that the submodular function related to the convex function by Lovász extension.

Lovász extension Given a set function $f$ with

$$
f(\phi)=0,
$$

the Lovász extension $\tilde{f}$ is defined as follows.

For $X \in R^{n}$, order the components in decreasing order $x_{i_{1}} \geq x_{i_{2}} \geq \cdots \geq x_{i_{n}}$,

$$
\tilde{f}(\boldsymbol{X})=\sum_{k=1}^{n} f\left(\left\{i_{1}, i_{2}, \cdots, i_{k}\right\}\right)\left(x_{i_{k}}-x_{i_{k+1}}\right)+f\left(\left\{i_{1}, i_{2}, \cdots, i_{n}\right\}\right) x_{i_{n}} .
$$

\section{Example B Lovász extension}

For set $\Omega=\{1,2\}$, its power set may be associated to a vertex of the hypercube $\{0,1\} \times\{0,1\}$ as follows

$$
\phi \rightarrow(0,0),\{1\} \rightarrow(1,0),\{2\} \rightarrow(0,1),\{1,2\} \rightarrow(1,1) .
$$

Now, consider set function $f$ with

$$
f(0,0)=f(1,1)=0, f(1,0)=f(0,1)=1 .
$$

Then its Lovász extension

$$
\tilde{f}\left(x_{1}, x_{2}\right)=\left|x_{1}-x_{2}\right|
$$

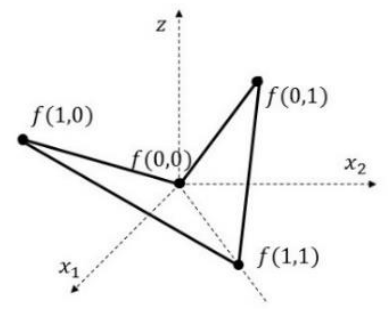

Figure 4. Lovász extension

From Figure 4. we understand that the image of extended function is two hyperplanes on $x_{1}>x_{2}$ and $x_{1}<x_{2}$, respectively.

Theorem A $[18,19]$ :

For set $\Omega=\{1,2\}$, without loss of generality assume the set function $f$ defined on vertices of the hypercube $\{0,1\} \times\{0,1\}$. Suppose $\tilde{f}$ is its Lovász extension. Then

(i) if $f$ is submodular function then $\tilde{f}$ is convex function,

(ii) $\min _{\left\{\delta_{i} \in\{0,1\}\right\}} f\left(\left\{\delta_{1}, \delta_{2}\right\}\right)=\min _{\left\{x_{i} \in[0,1]\right\}} \tilde{f}\left(\left\{x_{1}, x_{2}\right\}\right)$, (iii) the set of minimizers of $\tilde{f}$ on $[0,1] \times[0,1]$ is the convex hull of minimizers of $f$ on $\{0,1\} \times\{0,1\}$.

In the Example B, from Figure 4. we understand the extended function is convex function. In fact, $f$ is a submodular function with

$$
f(0,0)+f(1,1) \leq f(1,0)+f(0,1),
$$

and then the extended function $\tilde{f}$ is convex function by (i) of Theorem A.

In minimum norm methods we have to solve a discrete optimization problem (6)-(7). Benefiting from above Theorem with use of Lovász extension, we only need to solve one continuous convex problem. However, there are more convex optimal methods for continuous optimal problems compared to discrete ones. The solution of original problem will be given through simple process of projecting for the solution of extended problem.

\subsection{Continuous Energy Function for Proposed Method}

In equation (6) the wrap count $l_{i, j}$ can be arbitrary integer. But we can solve it step by step such that in every step $l_{i, j}$ can only take 0 or 1 [14]. The energy function with $L^{l}$ norm performs better than $L^{2}$ norm in when discontinuity preserving is concerned. In eq. (6) and (7), we take both data term and regularization term according to $L^{1}$ norm, then

$$
\begin{aligned}
& E=\sum_{i, j} E_{i, j}+\beta\left|l_{i, j}-l_{i-1, j}\right|+\text { verticalterm, } \\
& l_{i, j} \in\{0,1\},
\end{aligned}
$$

where

$$
a_{i, j}^{h}=\Delta^{h} \psi_{i, j}-W\left(\Delta^{h} \psi_{i, j}\right),
$$

is the jump count for wrapping point and

$$
E_{i, j}=E_{i, j}\left(l_{i, j}, l_{i-1, j}\right) \stackrel{\text { def }}{=} \sum_{i, j}\left|2 \pi\left(l_{i, j}-l_{i-1, j}\right)+a_{i, j}^{h}\right| .
$$

It should be emphasized that our regularization term changes the unwrapped function only by integer multiple of $2 \pi$ to satisfy congruent constraint. This is different with previous methods with regularization term.

In Lovász extension, the set function is required to satisfy eq. (9) that corresponding to

$$
E_{i, j}(0,0)=0
$$

Therefore, we replace $E_{i, j}\left(l_{i, j}, l_{i-1, j}\right)$ by $E_{i, j}\left(l_{i, j}, l_{i-1, j}\right)-E_{i, j}(0,0)$ in eq. (18). Since the function $E_{i, j}(0,0)$ is independent of variable $l_{i, j}$, this operation will preserve the same minimum point in eq. (16).

Now, by Lovász extension we have new continuous energy function as following

$$
\tilde{E}=\sum_{i, j} \tilde{E}_{i, j}+\beta\left|x_{i, j}-x_{i-1, j}\right|+\text { verticalterm, } x_{i, j} \in[0,1] \text {, }
$$

where 


$$
\tilde{E}_{i, j}= \begin{cases}\left(E_{i, j}(1,0)-E_{i, j}(0,0)\right)\left(x_{i, j}-x_{i-1, j}\right), & x_{i, j}>x_{i-1, j} \\ \left(E_{i, j}(0,1)-E_{i, j}(0,0)\right)\left(x_{i-1, j}-x_{i, j}\right), & \text { otherwise }\end{cases}
$$

Using the convexity of the $L^{1}$ norm, it is easily proved that the function $E_{i, j}$ satisfy equation (15) and is the submodular function.

By Theorem A, the extended function $\tilde{E}$ is convex function. Therefore, it is sufficient to solve a convex optimal problem with continuous energy function for unwrapping which can reach global minimum.

\subsection{Double Simple Averaging Subgradient Method}

Note that the function given by Lovász extension is nondifferential function. Then we can't use usual methods such as gradient method to minimize extended problem. Recently, many methods have been reported to solve minimization problem with non-differential function such as Chambolle's projection algorithm or other dual method [21]. However, compared with these methods, the subgradient method is very simple.

Specifically we use the double averaging subgradient method to solve the minimization problem which can make the iteration converge stably [20]. Using this method, the minimum point is given by iteration procedure as follows

$$
\boldsymbol{X}^{(n+1)}=\frac{n+1}{n+2} \boldsymbol{X}^{(n)}+\frac{1}{n+2}\left(\boldsymbol{X}^{(0)}-\alpha \sum_{k=0}^{n} \partial \tilde{E}\left(\boldsymbol{X}^{(k)}\right)\right), \quad n=0,1, \cdots
$$

where vectors $\boldsymbol{X}=\left\{x_{i, j}\right\}, \partial$ denote the subgradient of the function and $\alpha$ is the stepsize.

We leave the details of this method to Appendix A.

\subsection{Algorithm}

$$
\begin{aligned}
& \text { Initialization } l_{i, j}=\tilde{l}_{i, j}=\{\cdots, 0.5, \cdots\}, \\
& \text { improvement }=1
\end{aligned}
$$

\section{1 while improvement do}

2 Combining eqs. (20) and (21), compute the subgradient of energy functional $\tilde{E}$ with given $\boldsymbol{X}^{(0)}=\left\{l_{i, j}\right\}$.

3 Compute the minimal point $\left\{x_{i, j}^{\min }\right\}$ by iteration eq. (22).

4. let $\tilde{l}_{i, j}=l_{i, j}+\left[x_{i, j}^{\min }\right]_{+}$.

5 if $\tilde{E}\left(\tilde{l}_{i, j}\right)<\tilde{E}\left(l_{i, j}\right)$ then

$6^{l_{i, j}=\tilde{l}_{i, j}}$ go to step 2 .

else

7 improvement $=0$

8 end if

9 end while

In step 3, step size $\alpha$ is given by eq. (A3) (Appendix A) with

$$
M=0.5 * \sqrt{\text { image size }} .
$$

The projection

$$
[y]_{+}= \begin{cases}1, & y \geq 1 \\ 0, & y \leq 0 \\ \operatorname{int}(y+0.5), & \text { other wise. }\end{cases}
$$

used in step 4 , in order to solve integer minimum from the solution of extended continuous problem.

\section{EXPERIMENTS}

In this section we show some experiment results. We will compare the results of our algorithm against to the three typical methods those are Branch Cuts, weighted least-squares (PCG) and LP-PDE with $L^{1}$ norm. These algorithms are available at $\mathrm{ftp} / / / \mathrm{ftp}$.wiley.com/public/sci_tech_med/phase_unwrapping.

\subsection{Noise free images (Without regularization term)}

In this subsection some unwrapping results are given using noise free images In these simulations, we have used a set of 6 grayscale $321 \times 481$ shown in Figure5., these are available at Berkeley segmentation dataset [22]

https://www2.eecs.berkeley.edu/Research/Projects/CS/vision/bsds.

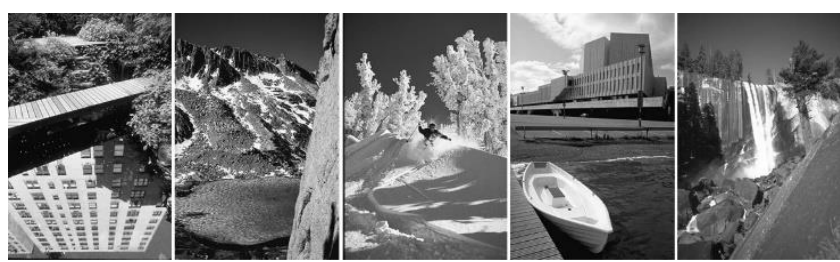

Figure 5. Test images. Bridge, Mountain, Ski, Boat, Falls

Note that the grey level of test images are rescaled into [0, $2.3 \pi]$ and multiplied a Gaussian function and then be wrapped into $[-\pi, \pi]$ by wrapping operation as in equation (1).

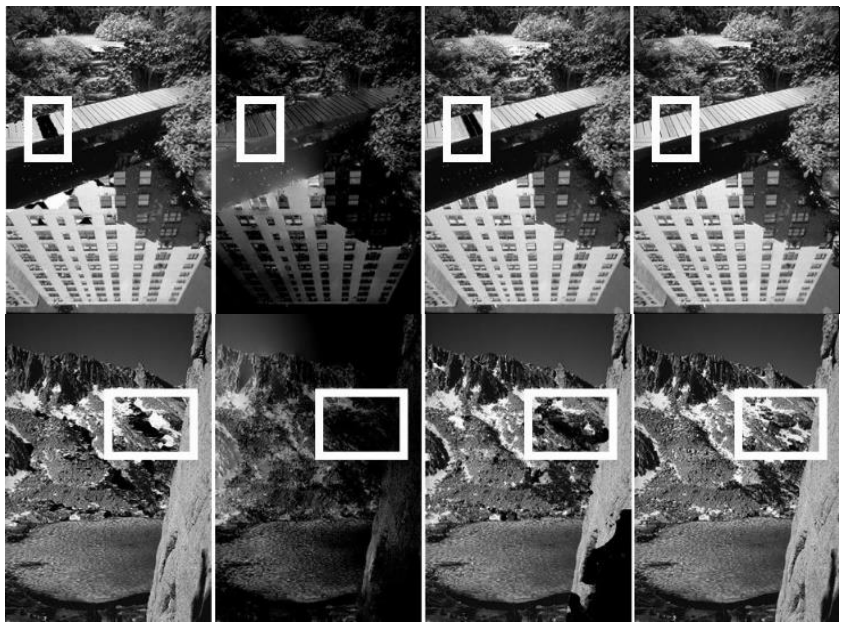

Figure 6. Unwrapping results for Bridge and Mountain. From left to right: Branch Cuts, PCG, LP-PDE, and proposed algorithm

Figure 6. shows unwrapping results with Bridge and Mountain image respectively. For Bridge image, some errors in bridge and building are produced by Branch Cuts method, while errors in bridge are produced by LP-PDE method respectively. The PCG 
method gives image of incorrect contrast. The proposed method recovered image almost exactly. Figure 6 . shows, the proposed method successfully unwraps of the Mountain image for which all other three methods produce some errors.

In Table 2. , for the quantitative comparison, we show the rootmean-square error (RMSE) defined by

$$
R M S E=\sqrt{\frac{\sum_{i, j}\left(\phi_{i, j}^{\text {unwrap }}-\varphi_{i, j}^{\text {correct }}\right)^{2}}{\text { image size }}},
$$

where $\phi_{i, j}^{\text {unwrap }}$ denotes the pixel value of unwrapped image and $\varphi_{i, j}^{\text {correct }}$ denotes the pixel value of correct image. Then we find that for all the six images, proposed method gives unwrapping images with smallest errors.

Table 2. Error of unwrapping results (RMSE)

\begin{tabular}{|c|c|c|c|c|}
\hline & $\begin{array}{c}\text { Branch } \\
\text { Cuts }\end{array}$ & PCG & $\begin{array}{c}\text { LP-PDE } \\
L^{1} \text { norm }\end{array}$ & $\begin{array}{c}\text { Proposed } \\
\text { algorithm }\end{array}$ \\
\hline Bridge & 0.95 & 2.91 & 0.76 & $\mathbf{0 . 3 0}$ \\
\hline Mountain & 1.59 & 2.78 & 2.18 & $\mathbf{0 . 7 3}$ \\
\hline Ski & 0.84 & 2.82 & 0.42 & $\mathbf{0 . 3 6}$ \\
\hline Boat & 0.70 & 2.97 & 1.37 & $\mathbf{0 . 3 5}$ \\
\hline Falls & 0.20 & 2.78 & 0.40 & $\mathbf{0 . 1 7}$ \\
\hline
\end{tabular}

\subsection{With regularization term for noisy images}

We also have done several simulations with regularization term with noisy images in order to illustrate the robustness of our algorithm to noise.

However, it is difficult to simply compare unwrapping images with noises. Hence, we will compare these corresponding label images instead. The label image is given by $2 \pi_{i, j}$ that is related to the unwrapped image as following

$$
\varphi_{i, j}=\psi_{i, j}+2 \pi l_{i, j}
$$

where $\left\{\varphi_{i, j}\right\}$ denotes the unwrapped image and $\left\{\psi_{i, j}\right\}$ denotes the wrapped (measured) image. Then unwrapping procedure is equivalent to solving correct label images.

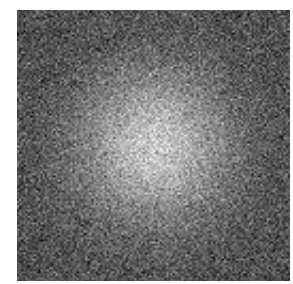

(a)

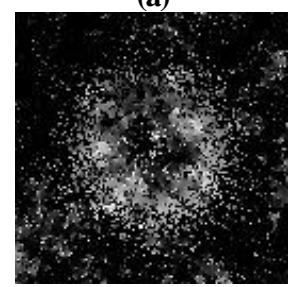

d)

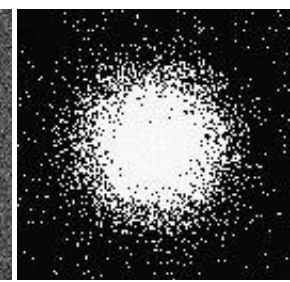

(b)

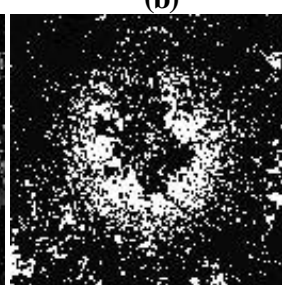

(e)

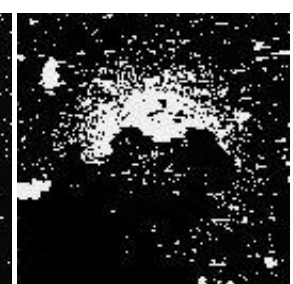

(c)

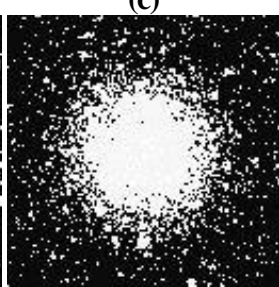

(f)

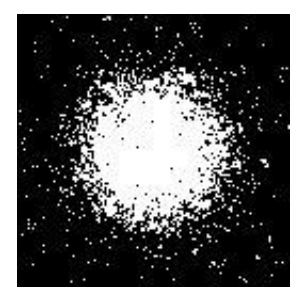

(g)

Figure 7. (a) Phase image with noise, (b) Correct Label image, (c) Branch-Cuts, (d) PCG, (e) LP-PDE ( $L^{1}$ norm), (f) Proposed algorithm without regularization term, (g) Proposed algorithm with regularization term

Figure 7. (a) shows original phase image (size: $128 \times 128)$ formed by Gaussian elevations with 8 height where Gaussian noise (the standard deviation 1.4)were added.

Figure 7. (b) shows correct label image. Figure 7. (c), (d), and (e) show unwrapped images by Branch-Cuts, PCG and LP-PDE ( $L^{1}$ norm) respectively. Compared with Figure 7. (b), these images are either incomplete or incorrect with making some additional holes. Figure 7. (f) and (g) show unwrapped images by proposed method. These two images are close to Figure 7. (b) which means the unwrapping is correct approximately.

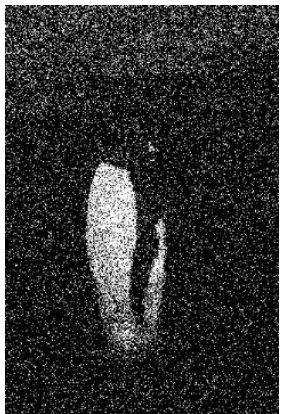

(a)

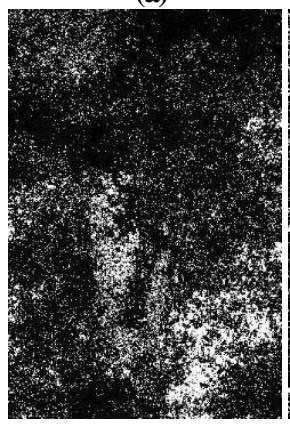

(d)

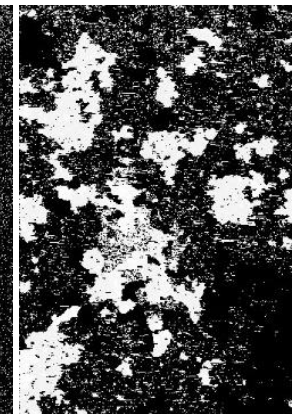

(b)

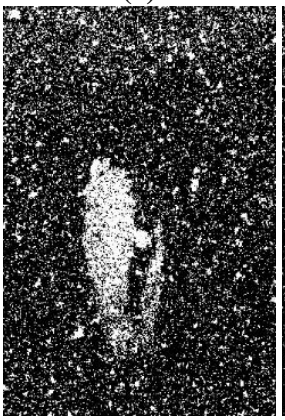

(e)

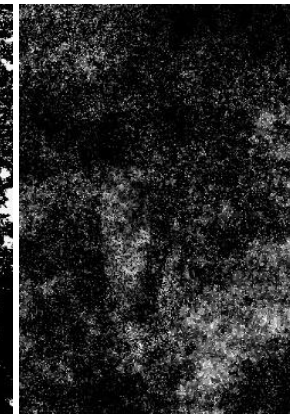

(c)

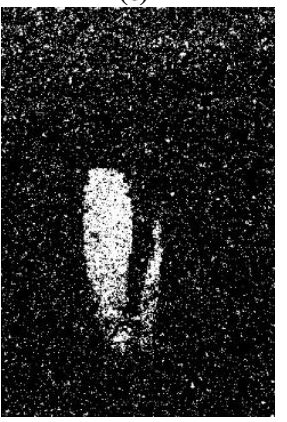

(f)
Figure 8. Penguin (a) Label image with true image, (b)

Branch-Cuts, (c) PCG, (d) LP-PDE ( $L^{1}$ norm), (e) Proposed algorithm without regularization term, (f) Proposed algorithm with regularization term

We also have done experiment with photo of a penguin. This image used in Figure 8. is available at the Berkeley segmentation dataset.

The noise is added to the image, after rescaling its grey level. Figure 8. (a) shows correct label image. Figure 8. (b), (c), and (d) are given by Branch-Cuts, PCG and LP-PDE ( $L^{1}$ norm) respectively. Thereby we can hardly see the penguin. Figure 8. (e) is given by proposed method without regularization term. Here the 
penguin is visible but affected by the noise greatly. Comparison with Figure 8. (f), we learn that our regularization term leads the image to prevent noises satisfactorily.

Note that in our compared experiments we skip two state of the art methods, the MCF in path-following and Graph-cuts method. These two methods can also reach global minimum of energy function with $L^{1}$ norm. Theorem $\mathrm{A}$ in section 3 ) guarantees that the proposed method without regularization term will give the same solution with those methods. That is the reason why corresponding comparison is skipped.

\section{CONCLUTION}

In this paper, we proposed a new minimum norm method for phase unwrapping. Benefiting from Lovász extension, it will solve the continuous energy minimization problem and can also reach a global optimal solution with congruent constraint (CC). This is the first result in phase unwrapping. Moreover, the Algorithm is very simply implemented and robust for noisy images. It can be also easily extended to the higher dimension situation to solve the $3 \mathrm{D}$ or $4 \mathrm{D}$ unwrapping problem. The simulation verified the effectiveness of the proposed method.

\section{Appendix A:}

\section{Double simple averaging subgradient method}

The vector $\mathbf{F}$ is subgradient of the function $g$ at $x_{0}$, if

$$
g(x) \geq g\left(x_{0}\right)+\boldsymbol{F}^{T}\left(x-x_{0}\right) \text { for all } x .
$$

When $g$ is differentiable, the only possible choice for $\boldsymbol{F}$ is $\nabla g\left(x_{0}\right)$. For non-differential function, the subgradient is not unique generally. Now, given non differential convex function $g$, we can solve its minimum by subgradient method as following

$$
\boldsymbol{X}^{(n+1)}=\boldsymbol{X}^{(n)}-\alpha \partial g\left(\boldsymbol{X}^{(n)}\right), \quad n=0,1, \cdots,
$$

where $\partial g$ is any subgradient of $g$ at $\mathbf{X}^{(n)}$ and $\mathbf{X}^{(0)}$ is initial value. Note that for nonsmooth functuions we cannot expect subgradients be vanishing in a neighborhood of the optimal solution. Hence the step size is given as

$$
\alpha=\frac{M}{L \sqrt{n+1}},
$$

wherer $M$ is an upper bound for initial point to the optimal solution and $L$ is given by

$$
\|\partial g\|_{2} \leq L,
$$

It is well known, for differential function, the subgradient method will reduce to the gradient method. But the subgradient method is not a descent method generally. In some steps, the subgradient $-\partial g\left(\mathbf{X}^{(n)}\right)$ may be ascent direction. That means

$$
g\left(\boldsymbol{X}^{(n+1)}\right)>g\left(\boldsymbol{X}^{(n)}\right),
$$

and results the convergence of subsequence becomeing unstable. This difficulty can be solved by using Double simple averaging subgradient method. In there the iteration procedure (A2) is replaced by following ones

$$
\boldsymbol{X}^{(n+1)}=\frac{n+1}{n+2} \boldsymbol{X}^{(n)}+\frac{1}{n+2}\left(\boldsymbol{X}^{(0)}-\alpha \sum_{k=0}^{n} \partial g\left(\boldsymbol{X}^{(k)}\right)\right), \quad n=0,1, \cdots,
$$

Theorem B [20]:
Suppose $g$ is a closed convex function and its domain $\mathrm{Q}$ is a closed convex set with smooth boundary. Assume that sequence $\left\{\boldsymbol{X}^{(n)}\right\}(n \geq 0)$ be generated by iteration (A6) for $\boldsymbol{X}^{(0)} \in \operatorname{int} Q$. Then

$$
\lim _{n \rightarrow \infty} g\left(\boldsymbol{X}^{(n)}\right)=\min _{Q} g(\boldsymbol{X}) \cdot
$$

\section{ACKNOWLEDGMENTS}

This work was partially supported by JSPS KAKENHI (Grant Number 15K06103) and the JST-ERATO project (Grant Number JPMJER1403).

\section{REFERENCES}

[1] Massonnet, D., and Feigl, K. L. 1998. Radar interferometry and its application to changes in the earth's surface. Rev. Geophys. 36(1998), 441-500.

[2] Rosen, P. A., Hensley, S., Joughin, I. R., Li, F. K., Madsen, S. N., Rodriguez, E., and Goldstein, R. 2000. Synthetic aperture radar interferometry. Proc. IEEE. 88(2000), 333-382.

[3] Pfeiffer, F., Weitkamp, T., Oliver, B., Christian, D. 2006. Phase retrieval and differential phase-contrast imaging with low-brilliance X-ray sources. Nature Phys. 2(2006), 258-261.

[4] Weitkamp, T., Diaz, A., David, C., Christian, D., Pfeiffer, F., Stampanoni, M., Cloetens, P., Ziegler, E. 2005. Quantitative $\mathrm{X}$-ray phase imaging with a grating interferometer. Opt. Express. 13(2005), 6296-6304.

[5] Jezzard, P., and Balaban, R. S. 1995. Correction for geometric distortion in echo planar images from B_0 field variations. Magnetic Resonance in medicine. 34(1995) 65-73.

[6] Itoh, K. 1982. Analysis of the phase unwrapping problem. Appl. Opt. 21(1982), 2470-2470.

[7] Flynn, T. 1997. Two-dimensional phase unwrapping with minimum weighted discontinuity. J. Opt. Soc. Amer. A. 14(1997), 2692-2701.

[8] Costantini, M. 1998. A novel phase unwrapping method based on network programing. IEEE Trans. Geosci. Remote Sens. 36(1998), 813-821.

[9] Ghiglia, D., and Pritt, M. 1998. Two-Dimensional Phase Unwrapping. Theory, Algorithms, and Software (New York: Wiley, 1998).

[10] Ghiglia, D.C., Romero, L.A. 1996. Minimum lp-norm twodimensional phase unwrapping. J. Opt. Soc. Am. A, 13(1996), $1-15$.

[11] Loecher, M., Schrauben, E., Johnson, K., and Wieben, O. 2016. Phase unwrapping in 4D MR Flow with a 4D singlestep Laolacian Algorithm. Journal of Magneloechertic Resonance Imajing. 43(2016), 833-842.

[12] Huang, Howard Y. H., Tian, L., Zhang, Z., Liu, Y., Chen, Z., and Barbastathis, G. 2012. Path-independent phase unwrapping using phase gradient and total-variation(TV) denoising. Opt. Express. 20(2012), 14075-14089.

[13] Kamilov, U. S., Papadopoulos, I. N., Shoreh, M. H., Psaltis, D., and Unser, M. 2015. Isotropic inverse-problem approach for two-dimensional phase unwrapping. J. Opt. Soc. Am. A. 32(2015), 1092-1100 (2015).

[14] Bioucas-Dias, J. M., and Valadão, G. 2007. Phase unwrapping via graph cuts. IEEE Trans. Image Process. 16(2007), $698-709$. 
[15] Campbell, N. D. F., Vogiatzis, G., Hernández, C., and Cipolla, R. 2010. Automatic 3D object segmentation in multiple views using volumetric graph-cuts. Image and Vision Computing, 28(2010), 14-25.

[16] Vogiatzis, G., Torr, P., and Cippola, R. 2005. Multi-view stereo via volumetric graph-cuts. In Proc. IEEE Conf. Computer Vision and Pattern Recognition (CVPR-2005), 391-399.

[17] Dong, J., Chen, F., Zhou, D., Liu, T., Yu, Z., and Y. Wang. 2017. Phase unwrapping with graph cuts optimization and dual decomposition acceleration for 3D high-resolution MRI data. Magn Reson Med 77(2017), 1353-1358.

[18] Lovász, L. 1983. Submodular functions and convexity. In Mathematical programming: The state of the art, A. Bachem, M. Grötschel, and B. Korte, eds., (Springer-Verlag, Berlin, 1983), 235-257.
[19] Bach, F. 2011. Learning with submodular functions: A convex optimization perspective. arXiv: 1111.6453 (2011).

[20] Nesterov, Y., and Shikhman, V. 2014. Convergent subgradient methods for nonsmooth convex minimization. tech. report, Université catholique de Louvain, Center for Operations Research and Econometrics (CORE), (2014).

[21] Chambolle, A., 2004. An algorithm for total variation minimization and applications. J. Math. Imag. Vis. 20(2004), 89-97.

[22] Martin, D., Fowlkes, C., Tal. D., and Malik, J. 2001. A database of human segmented natural images and its application to evaluating segmentation algorithms and measuring ecological statistics. In Proc. 8th Int. Conf. Comput. Vis. 2(2001), 416-423. 